\title{
Interaction des microondes avec les milieux vivants
}

\author{
A.J. BERTEAUD*
}

\begin{abstract}
RÉSUME
L'absorption des microondes et des hautes fréquences par les milieux vivants présente un caractère spécifique lié notamment à la caractéristique diélectrique des divers tissus (quantité et état de l'eau), à la profondeur de pénétration de l'onde, à la forme et aux dimensions du milieu. On définit classiquement un taux d'absorption spécifique (SAR) variable en fréquence et on montre que les normes d'exposition sont étroitement dépendantes du SAR. Ces diverses données biophysiques illustrent la complexité d'interprétation des effets biologiques décrits dans la littérature.
\end{abstract}

\section{ABSTRACT}

Microwave and radiofrequency absorption by living tissues presents a specificity associated to the dielectric properties of various tissues (water content), the penetration depth, the shape and size of the living media. A specific absorption rate (SAR) is generally considered which is dependent of the wave frequency, and the safety standards are a function of the SAR. These various biophysical data show the difficulty to account for the biological effects which are described in the literature.

Dans le spectre des rayonnements électromagnétiques non ionisants, ceux qui correspondent aux microondes et aux hautes fréquences (HF) nécessitent un examen particulier de l'interaction physique onde - milieu vivant dans la mesure où les longueurs d'onde associées (métriques et centimétriques) sont peu différentes des dimensions humaines. On comprend aisément que, dans ces conditions, l'intensité du couplage puisse varier très sensiblement avec la fréquence des ondes considérées. Nous passons en revue quelques manifestations de ces effets dimensionnels étant entendu, qu'au niveau moléculaire, les énergies d'activation associées à ces rayonnements en microondes restent très inférieures aux énergies de liaison moléculaire les plus faibles. Des descriptions plus complètes sont données par ailleurs $[2,3,7]$.

* Centre national de la recherche scientifique (CNRS), Groupe de recherche "Organisation moléculaire et macromoléculaire" (GR 35), 2, rue Henry Dunant, 94320 Thiais. 


\section{PROPRIÉTÉS DIÉLECTRIQUES D'UN TISSU VIVANT}

Les propriétés diélectriques dans le domaine des microondes sont assez largement dominées par celles de l'eau. On sait, en effet, que la molécule d'eau polaire peut s'orienter dans le champ électrique de l'onde et présente un effet de relaxation diélectrique qui est maximal aux fréquences microondes et HF. Cela signifie qu'à ces fréquences un tissu hydraté a des pertes diélectriques très fortes qui s'ajoutent aux pertes de conduction associées aux charges électriques présentes dans le tissu. Cette propriété rend compte de l'efficacité bien connue des enceintes microondes tant dans le domaine alimentaire que pour les applications industrielles. Elle explique aussi que l'échauffement par microondes de tissus fortement hydratés (peau, muscle, organes internes) est nettement plus intense que celui des milieux peu hydratés (os, graisse, par exemple).

Cependant, avant d'envisager cet aspect final du problème qu'est l'échauffement du tissu, il y a lieu de considérer dans quelle mesure l'onde pénètre dans celui-ci et quel est son taux d'absorption, toutes propriétés qui sont également plus ou moins liées à la teneur en eau du tissu [2].

\section{PÉNÉTRATION DE L'ONDE DANS LE TISSU}

Le passage d'une onde d'un milieu 1 à un milieu 2 est caractérisé par un cœfficient de réflexion qui explicite la fraction de la puissance réfléchie sur l'interface entre les deux milieux. Si les deux milieux 1 et 2 ont des propriétés diélectriques voisines, il y a peu de réflexion. Mais si l'onde passe d'un milieu peu absorbant (l'air par exemple) à un milieu présentant de fortes pertes diélectriques (la peau notamment), une fraction importante de la puissance incidente est réfléchie.

Par exemple, pour des fréquences comprises entre $100 \mathrm{MHz}$ et $10 \mathrm{GHz}$, une fraction de 50 à 70 pour cent de la puissance incidente est réfléchie par la peau. La fraction restante est transmise dans le tissu et va se propager avec une atténuation plus ou moins rapide suivant les pertes diélectriques du tissu. Là encore, la profondeur de pénétration est directement fonction de la teneur en eau. Pour un tissu peu hydraté tel que la graisse, cette profondeur peut varier de $30 \mathrm{~cm}$ pour une fréquence de $100 \mathrm{MHz}$ jusqu'à $3 \mathrm{~cm}$ pour $10 \mathrm{GHz}$. Pour un tissu assez hydraté tel que le muscle, la profondeur varie de $4 \mathrm{~cm}$ pour $100 \mathrm{MHz}$ jusqu'à $0,2 \mathrm{~cm}$ pour $10 \mathrm{GHz}$.

D'une façon plus générale, on a représenté sur la figure 1 à partir de données de la littérature [6] les variations de la profondeur de pénétration avec la fréquence de l'onde pour les deux classes de tissu vivant. $\mathrm{Si}$ aux fréquences $\mathrm{HF}$ (inférieures à $100 \mathrm{MHz}$ ) la pénétration est supérieure à une dizaine de centimètres, ce qui implique une pénétration pratiquement totale du corps humain, aux fréquences correspondant aux microondes (disons de 1 à $100 \mathrm{GHz}$ ) la pénétration est limitée à quelques centimètres et même beaucoup moins pour les fréquences les plus élevées. Dans ce dernier cas, les organes internes du corps humain sont assez efficacement protégés. 


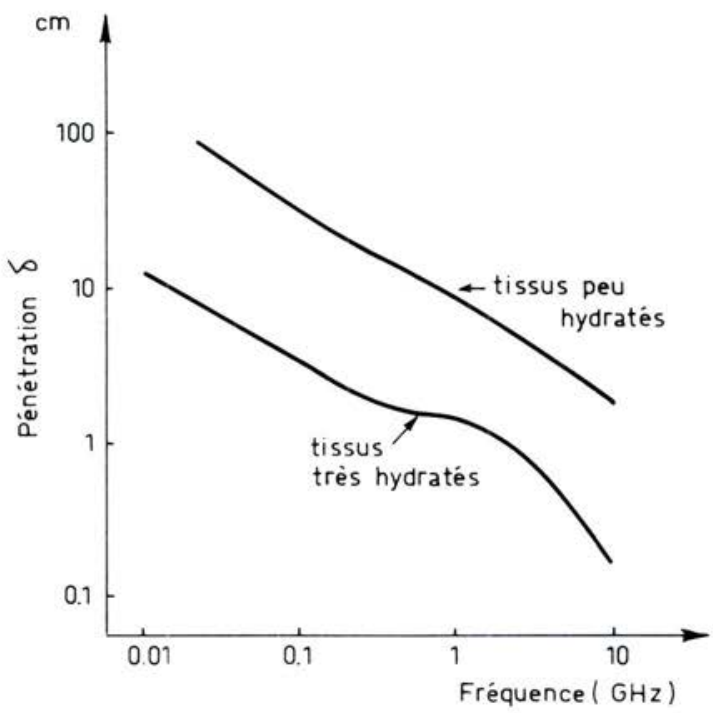

Fig. 1. - Profondeur de pénétration de tissus vivants en fonction de la fréquence de l'onde (d'après MYERS et al).

\section{ABSORPTION SPÉCIFIQUE EN FRĖQUENCES}

On a vu que les caractéristiques électriques d'un tissu, et notamment sa teneur en eau, sont telles que la fraction de puissance incidente qui est réfléchie à l'interface air-tissu et la profondeur de pénétration de l'onde sont directement fonction de sa fréquence et de la nature des tissus rencontrés. Une autre considération accroît la complexité du problème, à savoir la capacité d'absorption d'un milieu considéré globalement, tel que le corps humain, capacité qui peut varier dans de larges limites si ses dimensions sont de l'ordre de grandeur de la longueur d'onde associée au rayonnement. De nombreux calculs théoriques ont été réalisés pour déterminer l'absorption d'un ellipsoïde simulant le corps humain ou l'animal, placé dans diverses directions par rapport aux champs microondes. La capacité d'absorption est caractérisée par un paramètre désigné par SAR (specific absorption rate) et défini par la formule :

$$
\mathrm{SAR}=\frac{1}{2 \rho} \omega \varepsilon_{\mathrm{o}} \varepsilon^{\prime \prime} \mathrm{E}^{2}
$$

où $\rho$ représente la densité de masse, $\omega$ la pulsation de l'onde, $\varepsilon_{0}$ la permittivité du vide, $\varepsilon$ " les pertes diélectriques à la fréquence considérée, $E$ l'intensité du champ électrique interne. 


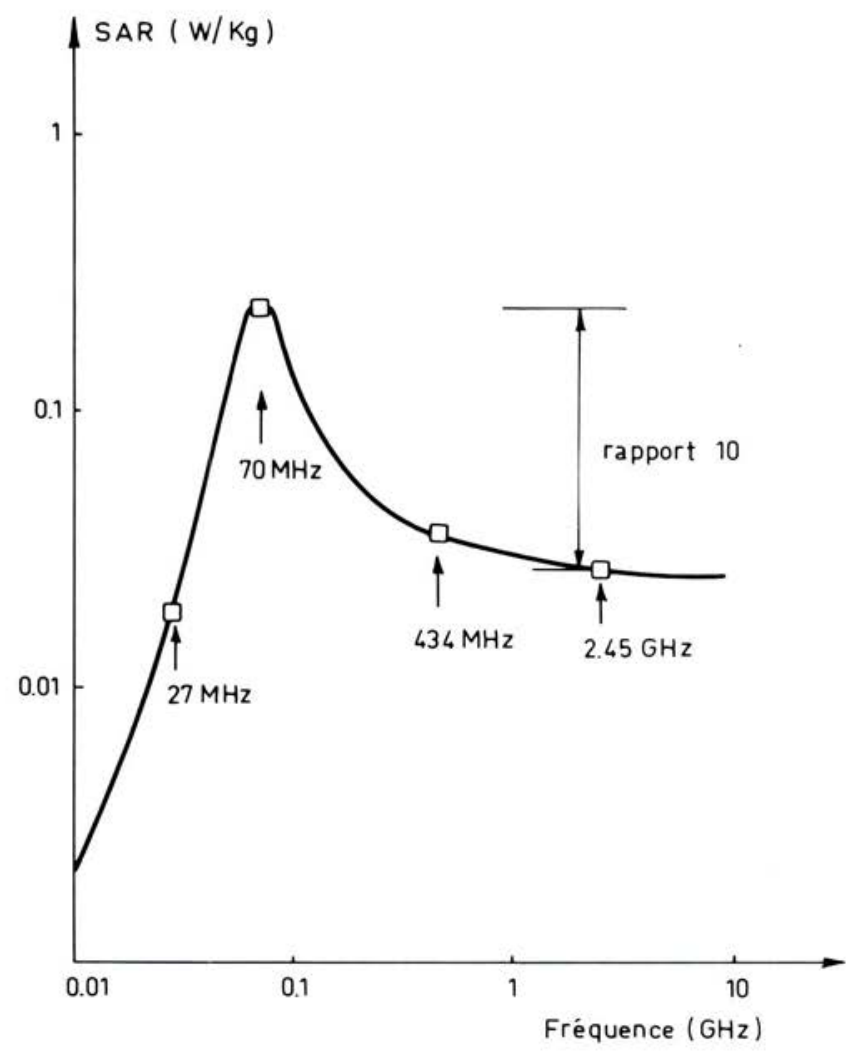

Fig. 2. - SAR d'un modèle simulant l'homme de $70 \mathrm{~kg}$ soumis à une densité de puissance de $1 \mathrm{~mW} / \mathrm{cm}^{2}$ (d'après DURNEY et al.) en fonction de la fréquence.

A partir des données de la littérature [4], on peut tracer, par exemple (fig. 2) les variations de SAR correspondant à un homme de $70 \mathrm{~kg}$ soumis à une densité de puissance constante, en fonction de la fréquence $(10 \mathrm{MHz}$ à $10 \mathrm{GHz}$ ). Cette courbe montre la présence d'une résonance dimensionnelle vers $70 \mathrm{MHz}$ et on observe qu'aux fréquences industrielles de $27 \mathrm{MHz}$, $434 \mathrm{MHz}$ ou $2,45 \mathrm{GHz}$, l'absorption de l'onde par le corps humain est 10 fois plus faible qu'à la fréquence de $70 \mathrm{MHz}$. Ces considérations d'absorption par résonance dimensionnelle jouent, évidemment, un grand rôle dans la définition des normes de sécurité. Par exemple, en portant sur un système d'axes analogue les variations en fréquences de la norme d'exposition maximale établie par l'American national standard institute (ANSI) [1] et de l'inverse du SAR, on obtient la figure 3 qui montre bien le parallélisme entre les valeurs de la norme et l'inverse de l'absorption. 


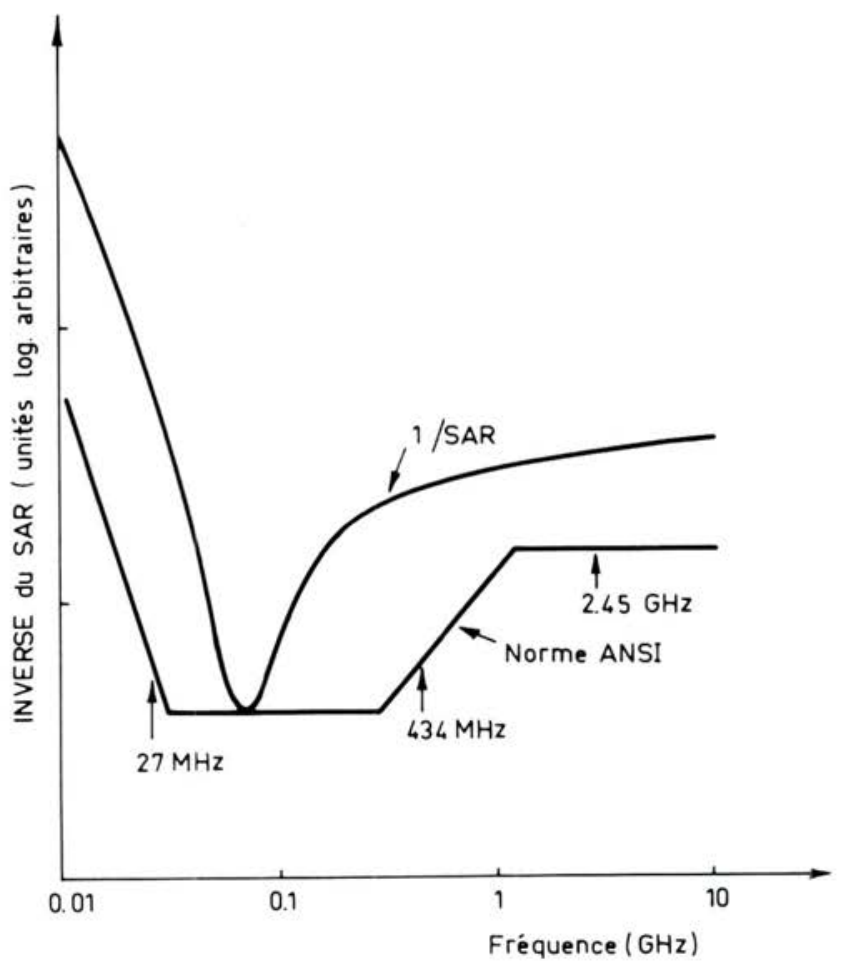

Fig. 3. - Variation comparée en fréquence de la norme américaine ANSI- C95 - 1 (1982) et de l'inverse du SAR représenté à la figure 2.

La vérification expérimentale des variations de SAR sur l'homme n'est pas réalisée pour l'instant et très peu d'études d'effets biologiques directes sur l'homme sont envisageables. On est donc conduit à extrapoler à l'homme l'observation d'effets biologiques étudiés sur l'animal, et l'existence des résonances dimensionnelles complique sérieusement le problème.

En effet, les dimensions de l'animal sont le plus souvent très différentes de celles de l'homme et les résonances propres sont alors déplacées. La figure 4 représente les variations en fréquence du SAR d'un homme de $70 \mathrm{~kg}$ et d'un rat de $300 \mathrm{~g}$ [4]. L'un résonne à $70 \mathrm{MHz}$ et l'autre vers $700 \mathrm{MHz}$. On voit qu'à $27 \mathrm{MHz}$, l'absorption d'un rat est environ 10 fois plus faible que celle de l'homme alors qu'à $2,45 \mathrm{GHz}$, l'absorption du rat est, au contraire, 10 fois plus forte. C'est dire que tel effet biologique observé sur un rat soumis à une densité de puissance de $10 \mathrm{~mW} / \mathrm{cm}^{2}$ nécessiterait pour être observée sur l'homme (en supposant toute chose égale par ailleurs) une densité de $1 \mathrm{~mW} / \mathrm{cm}^{2}$ seulement vers $27 \mathrm{MHz}$ et de 
$100 \mathrm{~mW} / \mathrm{cm}^{2}$ vers $2,45 \mathrm{GHz}$. Ces effets rendent compte, par exemple, des observations faites sur des rats à $2,45 \mathrm{GHz}[5]$.

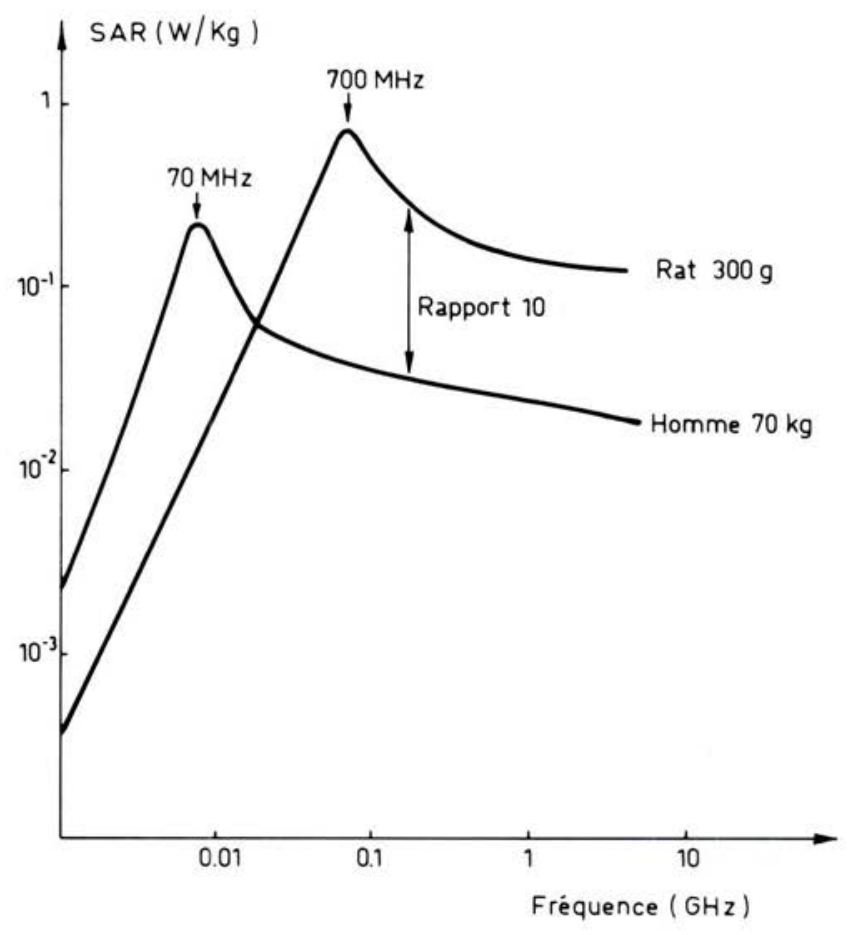

Fig. 4. - Variation comparée en fréquence du SAR d'un homme de $70 \mathrm{~kg}$ et d'un rat de $300 \mathrm{~g}$ soumis à une même densité de puissance de $1 \mathrm{~mW} / \mathrm{cm}^{2}$.

\section{CONCLUSION}

Dans le spectre des rayonnements électromagnétiques non ionisants, les fréquences correspondant aux microondes et aux hautes fréquences nécessitent une attention particulière sur le plan de la physique des interactions onde-matière par suite des similitudes entre les dimensions des longueurs d'ondes et celles des systèmes vivants que sont l'homme ou l'animal. Comme pour les autres domaines de fréquences hors des microondes (très basses fréquences, infra-rouge, ultra-violet, par exemple), des études d'effets biologiques sont cependant nécessaires pour fonder une normalisation de protection (voir l'article de M. DARDALHON p. 15). Mais l'interprétation et l'extrapolation à l'homme des résultats d'études biologiques faites sur l'animal restent très complexes et inachevées 
puisqu'elles doivent nécessairement tenir compte des interférences onde-matière et des résonances dimensionnelles propres à chaque espèce vivante.

\section{BIBLIOGRAPHIE}

[1] American National Standard Institute (ANSI) ANSI standard C95 - I, 1982 (Norme révisée).

[2] BERTEAUD A.J. Caractéristiques physiques des rayonnements électromagnétiques non ionisants. Ann. Ass. Belge de Radioprotection, 1981, 6, (1) 17-27.

[3] BERTEAUD A.J. Interactions of electromagnetic fields with living cells and molecular systems. In : Biological effects and dosimetry of non-ionizing radiation (GRANDOLFO M., MICHAELSON S. et RINDI A., Eds.). New York : Plenum Press NATO Series), $1983,319-335$.

[4] DURNEY C.H. et al. Radiofrequency radiation dosimetry handbook, 2. ed. USAF School of Aerospace medicine, 1978.

[5] KLEIN M.J., STERU L., MILHAUD C.L., ROSOLEN S.G. Application des techniques pharmacologiques à l'étude des effets biologiques des microondes. Radioprotection, 1982, 17, (4) 225-241.

[6] MYERS P.C., SADOWSKY N.L., BARRETT A.H. Microwave thermography ; principles, methods and clinical applications. J. Microwave Power, 1979, 14, (2) 105-115.

[7] STUCHLY M.A. Fundamentals of the interactions of radiofrequency and microwave energies with matter. In : Biological effects and dosimetry of nonionizing radiation (GRANDOLFO M., MICHAELSON S. et RINDI A., Eds.). New York: Plenum Press (NATO Series) 1983, 75-93. 